\title{
SOME BAZILEVIČ FUNCTIONS OF ORDER BETA
}

\author{
S.A. HALIM \\ Department of Mathematics and Computer Sciences \\ University of Wales \\ University College of Swansea \\ Singleton Park Swansea SA2, 8PP, U.K. \\ (Paper Received Apri1 4, 1988 and in Revised form September 13, 1988)
}

ABSTRACT. Distortion theorems and coefficient estimates are obtained for a new class of Bazilevic functions.

KEYWORDS AND PHRASES. BazileviC Functions, Functions Whose Derivative has Positive Real Part, Coefficient Estimates.

1980 AMS Classification Code. Primary $30 \mathrm{C} 45$.

1. INTRODUCTION.

Let $S$ be the class of normalized functions regular and univalent in the unit disc $D=z:\{|z|<1\}$ and $S^{*}$ the subclass of starlike functions. Denote by $P(\beta), t h e$ class of functions which are regular in $D$ and such that for $h \in P(B), h(0)=1$

and $\operatorname{Re} h(z)>B$ for $z \in D$. We write $P=P(0)$.

Bazilevic [1] showed that the class of normalized regular functions $f$ with representation

$$
f(z)=\left(\alpha \int_{0}^{z} p(t) g(t)^{\alpha} t^{-1} d t\right)^{\frac{1}{\alpha}}
$$

when $\alpha>0, g \in S^{*}$ and $p \in P$ for $z \in D$ forms a subclass of $S$. We denote this class of functions by $B(\alpha)$. See also [2].

Let $\alpha>0$. Then it follows easily from (1.1) that $f \in B(\alpha)$ if, and only if, there exists $g \in S^{*}$ such that for $z \in D$

$$
\operatorname{Re} \frac{z f^{\prime}(z)}{f(z)^{1-\alpha} \alpha_{(z)^{\alpha}}}>0
$$

In [3], Singh considered the subclass $B_{1}(\alpha)$ of $B(\alpha)$ obtained by taking $g(z) \equiv z$ in (1.2). Thus $f \in B_{1}(\alpha)$ if, and only if, for $\alpha>0$ and $z \in D$ 


$$
\operatorname{Re} \frac{z^{1-\alpha} f^{\prime}(z)}{f(z)^{1-\alpha}}>0
$$

We extend this class of functions as follows:

DEFINITION. Let $\mathrm{f}$ be regular in $\mathrm{D}$ with

$$
f(z)=z+\sum_{n=2}^{\infty} a_{n} z^{n}
$$

Then if $\alpha>0$ and $0<\beta<1, f \in B_{1}(\alpha, \beta)$ if, and only if, for $z \in D$

$$
\operatorname{Re} \frac{z^{1-\alpha} f^{\prime}(z)}{f(z)^{1-\alpha}}>B \text {. }
$$

We note that $B_{1}(1,0)=R$, the class of functions whose derivative has real part [4]. $B_{1}(1, \beta)$ was considered in [5]. Zamorski [6] and Thomas [7] solved the coefficient problem for $f \in B\left(\frac{1}{N}\right)$, in the case when $N$ is a positive integer. In [7], sharp distortion theorems were obtained for $f \in B_{1}(\alpha)$ for $\alpha>0$. The object of this paper is to extend these results to the class $B_{1}(\alpha, \beta)$. The class $B_{1}(\alpha, \beta)$ has also recently been considered in [8].

\section{RESULTS.}

\section{Distortion Theorems}

THEOREM 1. Let $\mathrm{f} \in \mathrm{B}_{1}(\alpha, \beta)$. Then for $z=r e^{i \theta} \in D, 0<r<1$,

$$
\begin{aligned}
& \text { (i) } Q_{2}(r)^{\frac{1}{\alpha}}<|f(z)|<Q_{1}(r)^{\frac{1}{\alpha}} \text {, } \\
& \text { (ii) if } 0<\alpha<1,
\end{aligned}
$$

$$
r^{\alpha-1} Q_{2}(r)^{\frac{1-\alpha}{\alpha}}\left(\frac{(1-r)(1-\beta)}{(1+r)}+\beta\right)<\left|f^{\prime}(z)\right|<r^{\alpha-1} Q_{1}(r)^{\frac{1-\alpha}{\alpha}}\left(\frac{(1+r)(1-\beta)}{(1-r)}+\beta\right)
$$

and if $\alpha>1$

$$
r^{\alpha-1} Q_{1}(r)^{\frac{1-\alpha}{\alpha}}\left(\frac{(1-r)(1-\beta)}{(1+r)}+\beta\right)<\left|f^{\prime}(z)\right|<r^{\alpha-1} Q_{2}(r)^{\frac{1-\alpha}{\alpha}}\left(\frac{(1+r)(1-\beta)}{(1-r)}+\beta\right)
$$

where

$$
Q_{1}(r)=\alpha \int_{0}^{r} \rho^{\alpha-1}\left(\frac{(1+\rho)(1-\beta)}{(1-\rho)}+\beta\right) d \rho,
$$

and 


$$
Q_{2}(r)=\alpha \int_{0}^{r} \rho^{\alpha-1}\left(\frac{(1-\rho)(1-\beta)}{(1+\rho)}+\beta\right) d \rho .
$$

Equality holds in all cases for the function $f_{\phi}$, defined by

$$
f_{\phi}(z)=\left(\alpha \int_{0}^{z} t^{\alpha-1}\left(\frac{\left(1+t e^{1 \phi}\right)(1-\beta)}{\left(1-t e^{1 \phi}\right)}+\beta\right) d t\right)^{\frac{1}{\alpha}}
$$

where $\phi=0$ or $\pi$.

PROOF.

(1) Since $f \in B_{1}(\alpha, \beta)$, and it follows from (1.4) that

$$
(1-\beta) p(z)=\frac{z^{1-\alpha} f^{\prime}(z)}{f(z)^{1-\alpha}}-\beta
$$

for $z \in D$ and $p \in P$.

Thus

$$
f(z)^{\alpha}=\alpha \int_{0}^{z} t^{\alpha-1}(p(t)(1-\beta)+\beta) d t
$$

and since $|p(z)|<\frac{1+r}{1-r}$ for $z \in D$, (see eg. [9]),

$$
\begin{aligned}
|f(z)|^{\alpha}<\alpha \int_{0}^{r} \rho^{\alpha-1} & \left(\frac{(1+\rho)(1-\beta)}{1-\rho}+\beta\right) d \rho \\
& =Q_{1}(r) .
\end{aligned}
$$

To obtain the left-hand inequality in (i), write

$$
h(z)=\frac{z^{1-\alpha_{f}}(z)}{f(z)^{1-\alpha}}
$$

Then (1.4) shows that $h \in p(\beta)$. Thus from, [5] (Theorem 1 with $c=1-2 \beta$ and $n=1$ ), we obtain

$$
\frac{(1-r)(1-\beta)}{(1+r)}+\beta<|h(z)|<\frac{(1+r)(1-\beta)}{(1-r)}+\beta .
$$

Hence from (2.3) and (2.4) we have

$$
\left|\frac{d}{d z}[f(z)]^{\alpha}\right|>\alpha r^{\alpha-1}\left(\frac{(1-r)(1-\beta)}{(1+r)}+\beta\right)
$$

Now let $z_{1},\left|z_{1}\right|=r$ be chosen so that $\left|f\left(z_{1}\right)^{\alpha}\right|<\mid f(z)$ q for all z with $|z|=r$. Writing $\omega=f\left(z_{1}\right)^{\alpha}$, it follows that since $f$ is univalent, the line segment $\lambda$ from 0

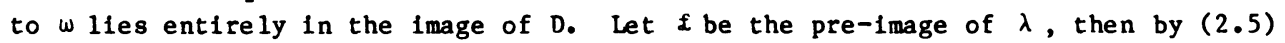




$$
\begin{aligned}
|f(z)|^{\alpha} \geqslant\left|f\left(z_{1}\right)\right|^{\alpha} & =\int_{\lambda}|d \omega|=\int_{L}\left|\frac{d \omega}{d z_{1}}\right|\left|d z_{1}\right| \\
& \geqslant \int_{0}^{r} \alpha \rho^{\alpha-1}\left(\frac{(1-\rho)(1-\beta)}{(1+\rho)}+\beta\right) d \rho
\end{aligned}
$$

which is the left-hand inequality in (i).

(ii) From (2.3) we have for $z=r e^{i \theta}$

$$
\left|f^{\prime}(z)\right|=r^{\alpha-1}|f(z)|^{1-\alpha}|h(z)|
$$

if $0<\alpha<1$, the inequalities follow at once from (2.6), (2.4) and (1). If $\alpha>1$, (1) gives

$$
Q_{1}(r)^{\frac{1-\alpha}{\alpha}}<|f(z)|^{1-\alpha} \leqslant Q_{2}(r)^{\frac{1-\alpha}{\alpha}} .
$$

Applying (2.4) and (2.7) to (2.6) gives the required result. Equality is attained in and (i) for $f_{0}$ and in (ii) for $f_{0}$ when $0<\alpha<1$ and for $f_{\pi}$ when $\alpha>1$.

The following shows that as $\alpha+0$ the bounds in Theorem 1 are asymptotic to the distortion theorems for starlike functions of order $B>0$ (see eg. [9]).

THEOREM 2. For $0<r<1$, let $Q_{1}(r)$ and $Q_{2}(r)$ be defined as in Theorem 1 . Then as $\alpha+0$

$$
\begin{aligned}
& \text { (i) } Q_{1}(r)^{\frac{1}{\alpha}} \sim \frac{r}{(1-r)^{2(1-\beta)}}, \\
& \text { (ii) } Q_{2}(r)^{\frac{1}{\alpha}} \sim \frac{r}{(1+r)^{2(1-\beta)}}, \\
& \text { (iii) } Q_{1} \text { (r) } \sim Q_{2}(r) \sim 1 .
\end{aligned}
$$

PROOF.

We prove ( 1 ), since (i1) and (i1i) are similar.

As $\alpha+0$,

$$
\begin{aligned}
& Q_{1}(r)^{\frac{1}{\alpha}}=\alpha \int_{0}^{r} \rho^{\alpha-1}\left(\frac{(1+\rho)(1-\beta)}{1-\rho}+\beta\right) d \rho \\
& =r\left(1+2 \alpha(1-\beta) r^{-\alpha} \int_{0}^{r} \frac{\rho^{\alpha}}{1-\rho} d \rho\right)^{\frac{1}{\alpha}} \\
& \sim r\left(1-2 \alpha(1-\beta) r^{-\alpha} \log (1-r)\right)^{\frac{1}{\alpha}} \\
& \sim r e^{-2(1-\beta) \log (1-r)}=\frac{r}{(1-r)^{2(1-\beta)}} .
\end{aligned}
$$

COROLLARY.

Suppose that $f(z) \neq w$ for $z \in D$, then 


$$
|\omega|>Q_{2}(1)^{\frac{1}{\alpha}} \sim 4^{\beta-1} \text { as } \alpha+0 .
$$

PROOF .

Let $a>0$, and $w$ be a point on the boundary of $f(D)$ closest to the origin. Let $L_{1}$ denote the straight line from 0 to $w$ and $L$ its pre-image in $D$. Then $|w|>|F(z)|$ for $z \in L \cap D$. Since the circle $|z|=r$ intersects $L$, at least

once, Theorem 1 (i) gives $|\omega|>Q_{2}(r)^{\frac{1}{\alpha}}$.

Thus Theorem 2 (ii) gives

$$
|\omega|>Q_{2}(1)^{\frac{1}{\alpha}} \sim 4^{\beta-1} \text { as } \alpha+0 \text {. }
$$

3. A COEFFICIENT THEOREM

Notation: ${ }_{n}^{\infty}=0$

THEOREM 3. Let $f \in B_{1}\left(\frac{1}{N}, \beta\right)$ and be given by (1.3) where $N$ is a positive integer. Suppose also that for $z \in D$,

$$
f_{0}(z)=z+\sum_{n=0}^{\infty} \gamma_{n} z^{n} \text { where } f_{0}(z) \text { is given by }(2.1) \text {. }
$$

Then (i) $f(z) \ll f_{0}(z)$,

and (ii) $\gamma_{n} \sim\left(\frac{2(1-B)}{N}\right)^{N}\left(\frac{N}{n}\right)(\log n)^{N-1}$ as $n+\infty$.

PROOF.

(i) Thomas [7], proved that if $\left|\alpha_{n}\right| \leqslant\left|\beta_{n}\right|$, then for $m=1,2,3, \ldots$,

$$
\left(\sum_{n=1}^{\infty} \alpha_{n} z^{n}\right)^{m} \ll\left(\sum_{n=1}^{\infty} B_{n} z^{n}\right)^{m} \text {. }
$$

Write $p(z)=1+\sum_{k=1}^{\infty} p_{k} z^{k}$. Then (2.2) gives

$$
\begin{gathered}
f(z)^{\frac{1}{N}}=\frac{1}{N} \int_{0}^{z} t^{\alpha-1}\left[\left(1+\sum_{k=1}^{\infty} p_{k} t^{k}\right)(1+\beta)+\beta\right] d t \\
=\frac{1}{N}\left[N(1-B) z^{\frac{1}{N}}+(1-\beta) \sum_{k=1}^{\infty}\left(\frac{P_{k} z^{k+\frac{1}{N}}}{k+\frac{1}{N}}\right)+B N z^{\frac{1}{N}}\right] \\
=z^{\frac{1}{N}}\left(1+\frac{(1-\beta)}{N} \sum_{k=1}^{\infty} \frac{P_{k} z^{k}}{\left(k+\frac{1}{N}\right)}\right) .
\end{gathered}
$$

Thus

$$
f(z)=z\left(1+\frac{(1-\beta)}{N} \sum_{k=1}^{\infty} \frac{P_{k} z^{k}}{\left(k+\frac{1}{N}\right)}\right)^{N}
$$


and since $p \in P$, we have $\left|P_{k}\right|<2[6]$. Hence

$$
\left.f(z)=z\left(1+\frac{(1-\beta)}{N} \sum_{k=1}^{\infty} \frac{P_{k} z^{k}}{\left(k+\frac{1}{N}\right)}\right)^{N} \| z_{i} 1+\frac{(1-\beta)}{N} \sum_{k=1}^{\infty} \frac{2 z^{k}}{\left(k+\frac{1}{N}\right)}\right)^{N}=f_{0}(z) .
$$

(ii) Putting $\alpha=\frac{1}{N}$ in $(2 \cdot 1)$, we have

$$
\begin{aligned}
f_{0}(z) & =z+\sum_{n=2}^{\infty} \gamma_{n} z^{n}=z\left(1+\frac{2(1-\beta)}{N} \sum_{n=1}^{\infty} \frac{z^{n}}{\left(n+\frac{1}{N}\right)}\right)^{N} \\
& =z \sum_{v=0}^{\infty}\left(\begin{array}{l}
N \\
v
\end{array}\right)\left(\frac{2(1-\beta)}{N}\right)^{\nu}\left(\sum_{n=1}^{\infty} \frac{z^{n}}{\left(n+\frac{1}{N}\right)}\right)^{\nu} \cdot
\end{aligned}
$$

Let

$$
\left(\sum_{n=1}^{\infty} \frac{z^{n}}{\left(n+\frac{1}{N}\right)}\right)^{\nu}=\sum_{n=\nu}^{\infty} D_{n}(v)_{z} n \quad(\nu=0,1,2,3, \ldots) .
$$

Thomas [7] proved that $D_{n}(\nu) \sim \frac{\nu}{N}(1 \circ \mathrm{n})^{\nu-1}$ as $n \rightarrow \infty$ and so this gives

$$
\begin{gathered}
\gamma_{n}=\sum_{\nu=0}^{\infty}\left(\begin{array}{l}
N \\
\nu
\end{array}\right)\left(\frac{2(1-\beta)}{N}\right)^{\nu} D_{n}(\nu) \\
\sim\left(\frac{2(1-\beta)}{N}\right)^{N}\left(\begin{array}{l}
N \\
n
\end{array}\right)(1 \circ \mathrm{log})^{N-1} \text { as } n+\infty .
\end{gathered}
$$

\section{REFERENCES}

1. BAZILEVIČ, I.E., On a case of integrability in quadratures of the LoewnerKufarev equation, Mat. Sb. 37(79) (1955), 471-476.

2. THOMAS, D.K., On Bazilevtč functions, Math, Z. 109(1969), 344-348.

3. SINGH, R., On Bazilevič functions, Proc. Ame r. Mat. Soc. 38(1973), 261-271.

4. MACGREGOR, T.H., Functions whose derivative has positive real part, Trans. Ame r. Math. Soc. 104 (1962), 532-537.

5. TONTI, NORMAN E. \& TRAHAN, DONALD H., Analytic functions whose real parts a re bounded below, Math. Z. 115(1970), 252-258.

6. ZAMORSKI, J., On Bazilevič Schlicht functions, Ann. Polon. Math. 12 (1962), 8390.

7. THOMAS, D.K., On a subclass of Bazilevič functions, Internat. J. Math. and Math. Sc1., Vol.8, No. 4 (1985), 799-783.

8. HALIM, ABDUL S., On the coefficients of some Bazilevic functions of order $B$.. To appear.

9. Goobman, A.W., Univalent functions, Vol.I ., Mariner Publishing Co., Tampa Florida, 1983.

10. POMMERENKE, Ch., Univalent functions, Vandenhoeck and Ruprecht, Göttingen, 1975. 


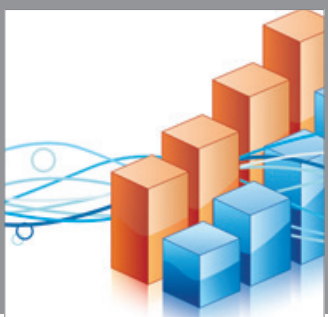

Advances in

Operations Research

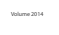

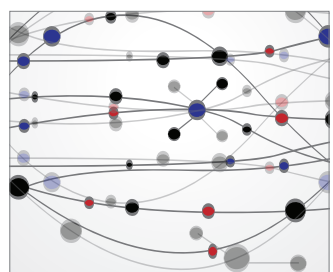

\section{The Scientific} World Journal
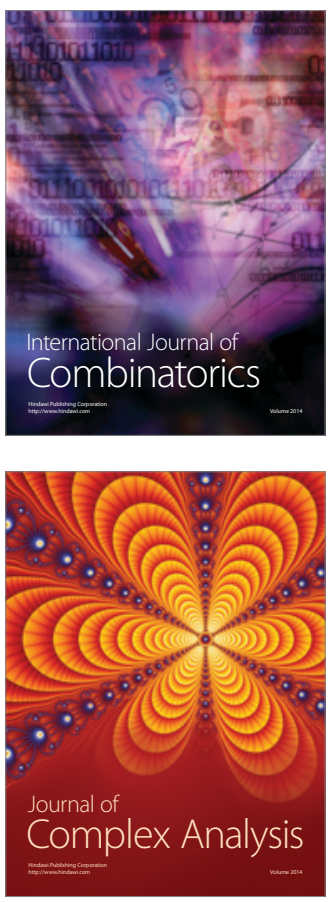

International Journal of

Mathematics and

Mathematical

Sciences
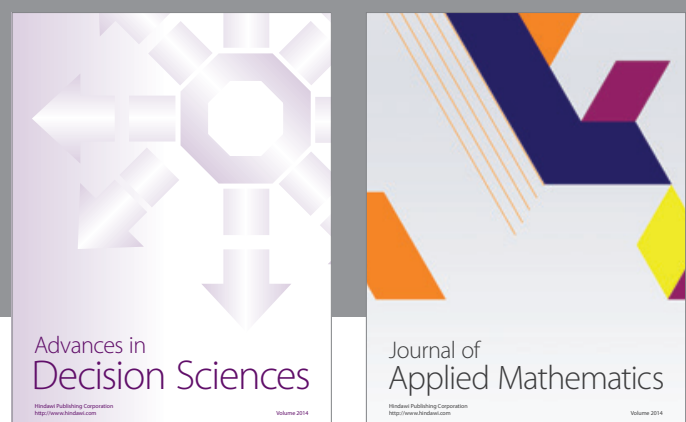

Journal of

Applied Mathematics
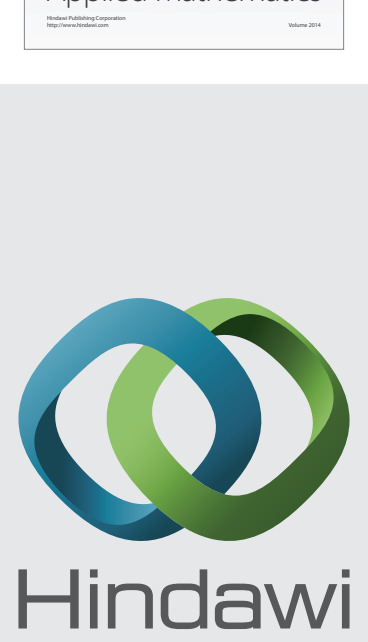

Submit your manuscripts at http://www.hindawi.com
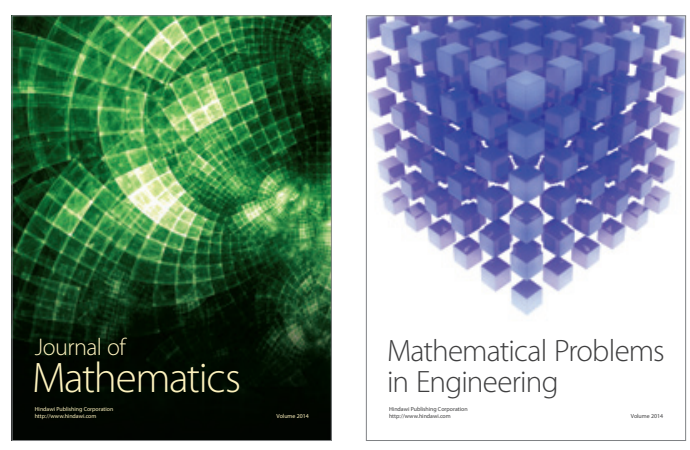

Mathematical Problems in Engineering
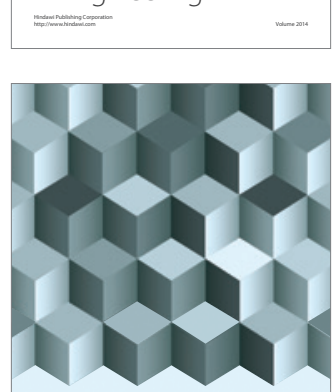

Journal of

Function Spaces
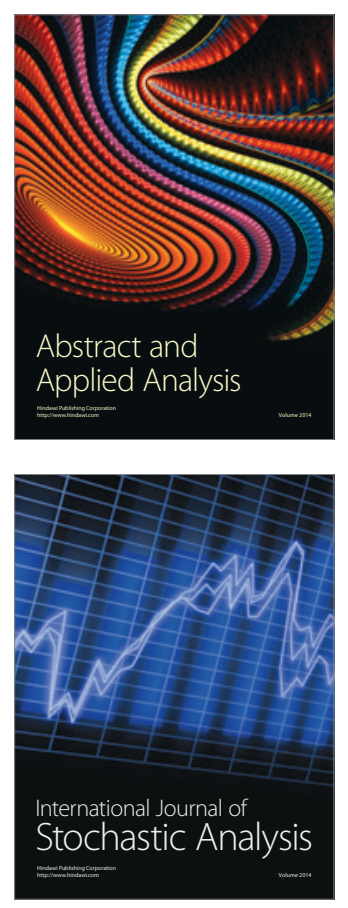

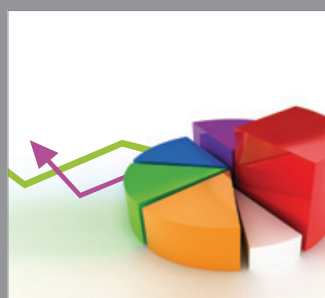

ournal of

Probability and Statistics

Promensencen
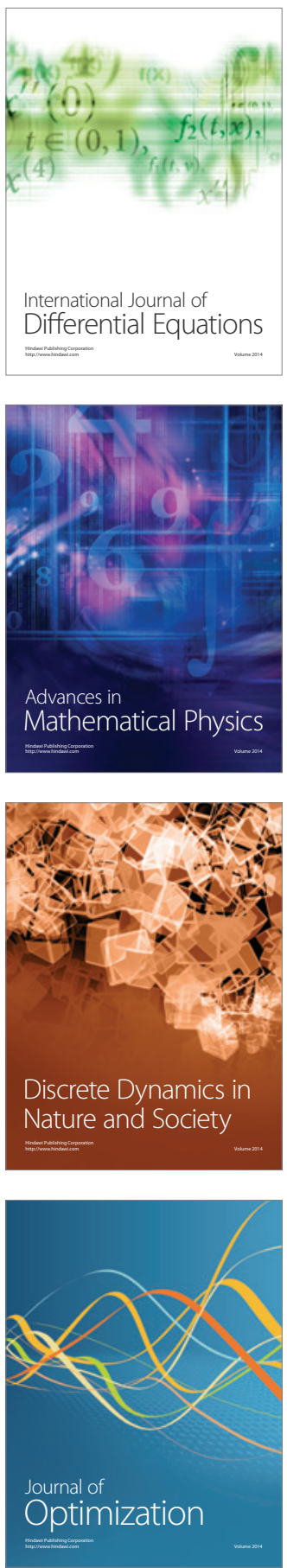